\title{
Immunotherapy of lymphomas
}

\author{
Stephen M. Ansell and Yi Lin \\ Department of Hematology, Mayo Clinic, Rochester, Minnesota, USA.
}

\begin{abstract}
Lymphoid malignancies typically promote an infiltrate of immune cells at sites involved by the disease. While some of the immune cells present in lymphoma have effector function, the immune system is unable to eradicate the malignant clone. Therapies that optimize immune function therefore have the potential to improve the outcome of lymphoma patients. In this Review, we discuss immunologic approaches that directly target the malignant cell as well as approaches to optimize both the innate and adaptive immune response to the tumor. While many of these therapies have shown single-agent activity, the future will clearly require thoughtful combinations of these approaches.
\end{abstract}

\section{Introduction}

Lymphomas are malignancies of lymphocytes in which the malignant cell recruits and sustains a microenvironment of other immune cells as well as stromal elements that promote malignant cell growth and survival (1). While some immune cells may be part of an antitumor immune response, many cells in the microenvironment suppress immune function (2). The tumor microenvironment differs between different types of lymphoma, ranging from a highly inflamed environment such as that seen in Hodgkin lymphoma to a very anergic and immune-suppressed environment such as that seen in chronic lymphocytic leukemia (CLL) (3). Some tumor microenvironments have a preponderance of $\mathrm{T}$ cells, such as that seen in follicular lymphoma, while others have a preponderance of macrophages, such as that seen in Burkitt lymphoma (3). While much research continues to determine the relative roles of cell populations in the lymphoma microenvironment and to identify critical pathways responsible for effective immune cell function, clinical trials have tested agents and strategies that utilize the immune system to target and suppress the malignant clone. In this Review, we summarize the clinical results with agents that directly target the malignant cell and utilize the immune system for effector function, as well as antibodies that deliver toxic payloads to the malignant cell. We also review immunotherapies that target nonmalignant immune cells in the tumor to activate them and thereby promote an antitumor immune response, including immune checkpoint-blocking antibodies and vaccine approaches. Finally, we review results from clinical trials using chimeric antigen receptor (CAR) $\mathrm{T}$ cells that ensure immune engagement with the malignant cell, as well as immunomodulatory drugs that change the composition of the tumor microenvironment (Figure 1). While many of these approaches are effective as a single-agent

Conflict of interest: SMA received research funding (to his institution) for clinical trials from Bristol-Myers Squibb, Seattle Genetics, Takeda, Regeneron, AI Therapeutics, Affimed, and Trillium. YL received research funding to her laboratory from Janssen and research funding (to her institution) for clinical trials from Kite/Cilead, Bluebird Bio, Celgene, Janssen, Merck, and Takeda.

Copyright: (5) 2020, American Society for Clinical Investigation.

Reference information: J Clin Invest. 2020;130(4):1576-1585.

https://doi.org/10.1172/JCI129206. strategy, the future clearly will lie in combining approaches to improve patient outcomes.

\section{Monoclonal antibodies}

Antibodies targeting cell surface receptors have become a mainstay of therapy in cancer treatment. In lymphoma, initial studies targeted CD20 using a chimeric monoclonal antibody, rituximab. Subsequent trials have targeted other cell surface receptors on the malignant cell or have focused on modifying the Fc portion of the antibody to engage the immune system, specifically macrophages and other phagocytic cells, more effectively (Table 1). More recently, monoclonal antibodies have been generated that target receptors on immune cells, either to prevent inhibition of their function by immunosuppressive ligands or to directly stimulate the cell by engaging activating receptors in an agonistic fashion.

Targeting malignant cells. Initial monoclonal antibody approaches targeted CD20, and the first studies used a chimeric monoclonal antibody, rituximab $(4,5)$. Rituximab showed significant single-agent activity in the relapsed setting in indolent lymphoma and rapidly became standard therapy in both the relapsed and the front-line setting either as a single agent or in combination with other agents, including chemotherapy. Treatment with rituximab impacted not only progression-free survival (PFS) but overall survival as well, and rituximab has become a standard therapy in most B cell malignancies $(6,7)$. Next, rituximab was combined with other monoclonal antibodies targeting cell surface receptors on the malignant B cell. The combination of an anti-CD20 antibody with antibodies targeting CD22 or CD80 also resulted in high response rates, particularly in follicular lymphoma $(8,9)$.

Following the success of rituximab, a multitude of other anti-CD2O antibodies were developed. These antibodies either targeted a different epitope on CD20 or modified the structure of the monoclonal antibody to promote greater complementdependent or antibody-dependent cytotoxicity. The most promising of these has been obinutuzumab, a glycoengineered type II monoclonal antibody directed against CD20 that displays greater antibody-dependent cellular cytotoxicity. Obinutuzumab, when combined with chemotherapy, was found to be superior to rituximab-based chemoimmunotherapy in follicular lymphoma and small lymphocytic lymphoma/CLL (10-12). This was shown 


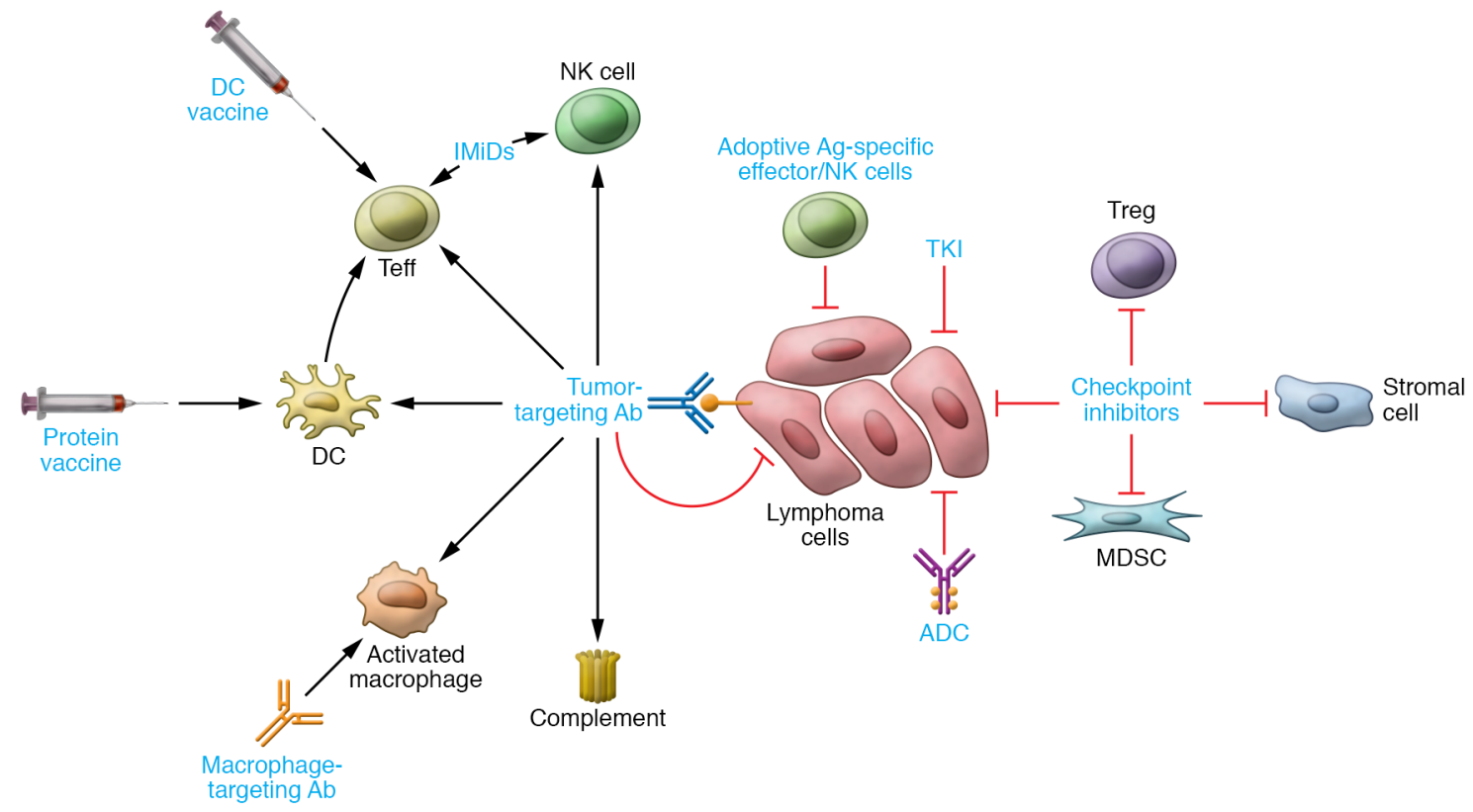

Figure 1. Overview of immunotherapy in lymphoma. ADC, antibody-drug conjugate; Ag, antigen; $D C$, dendritic cell; IMiDs, immunomodulatory drugs; MDSC, myeloid-derived suppressor cell; Teff, effector T cell; TKI, tyrosine kinase inhibitors; Treg, regulatory T cell. This figure was adapted from an image created by Arushi Khurana using BioRender.

to be true in relapsed patients refractory to rituximab, as well as in treatment-naive patients. Unfortunately, obinutuzumab has shown less promise in aggressive lymphomas such as diffuse large B cell lymphoma (DLBCL), in which its combination with chemotherapy has not proven superior to rituximab combined with chemotherapy (13).

Other unconjugated monoclonal antibodies targeting proteins on lymphoma cells have also proven promising, and this has included targets on both malignant B cells and T cells. MOR-208, which targets CD19 on malignant B cells, has shown particularly promising overall response rates when combined with the immunomodulatory drug lenalidomide in relapsed or refractory DLBCL. The overall response rates, complete response rates, and PFS seen with this combination in relapsed and refractory DLBCL have been superior to those seen with most other regimens in this setting (14). This has resulted in the MOR-28/lenalidomide combination receiving breakthrough designation from the US FDA. Similarly, mogamulizumab is a first-in-class defucosylated anti-CCR4 antibody with enhanced antibody-dependent cellular toxicity that has been evaluated in T cell lymphomas (15-17). CCR4 is a chemokine that is commonly expressed on tumor cells in T cell lymphomas, including cutaneous T cell lymphomas (CTCLs) (16, 17). Mogamulizumab was compared with vorinostat in a randomized trial of relapsed and refractory CTCL patients, and treatment with mogamulizumab resulted in more durable responses and a longer PFS (17). Based on these data, mogamulizumab was approved by the FDA for use in patients with relapsed and refractory CTCL.

Attempts to improve on the efficacy of monoclonal antibodies have subsequently included the development of antibody-drug conjugates (ADCs), in which monoclonal antibodies are engineered to deliver a toxic payload. The first ADC to demonstrate clinical activity was brentuximab vedotin, which uses an anti-
CD30 monoclonal antibody bound to an auristatin derivative, monomethyl auristatin E (MMAE), that disrupts microtubule function and inhibits cell division. Excellent response rates have been reported in both relapsed and refractory patients with Hodgkin lymphoma (HL) and when brentuximab vedotin is combined with standard chemotherapy as first-line therapy $(18,19)$. Similar impressive activity has been reported in patients with relapsed anaplastic large-cell lymphoma (ALCL) $(20,21)$. In a randomized trial with patients with relapsed cutaneous ALCL, brentuximab vedotin has been shown to be superior to standard treatment (22). In patients with systemic ALCL or other CD30-positive T cell lymphomas, the addition of brentuximab vedotin to standard frontline chemotherapy prolonged survival when compared with standard chemotherapy alone. This outcome set a new standard for the treatment of CD30-positive T cell lymphomas (23).

Using the same linker and toxin as brentuximab vedotin, polatuzumab vedotin has been developed to target CD79b on malignant B cells. When combined with rituximab, polatuzumab vedotin demonstrated impressive response rates and a promising PFS in patients with follicular lymphoma and DLBCL (24). In a subsequent randomized trial, the addition of polatuzumab vedotin to bendamustine and rituximab combination therapy (BR) resulted in a superior median PFS and overall survival in relapsed DLB$\mathrm{CL}$ when compared with $\mathrm{BR}$ alone. However, this benefit was not seen in follicular lymphoma patients (25). This benefit to DLBCL patients resulted in the combination receiving breakthrough designation from the FDA, and there is currently a randomized phase III trial in progress evaluating the addition of polatuzumab vedotin to rituximab, cyclophosphamide, doxorubicin, and prednisone combination therapy (R-CHP) compared with rituximab, cyclophosphamide, doxorubicin, vincristine, and prednisone (R-CHOP) as initial therapy for DLBCL (NCT03274492, ClinicalTrials.gov). 
Table 1. Selected therapeutic targets on tumor cells evaluated in Iymphoma

\begin{tabular}{lcc}
$\begin{array}{l}\text { Therapy } \\
\text { Monoclonal antibodies }\end{array}$ & Target & \multicolumn{1}{c}{ Histology } \\
$\begin{array}{l}\text { Rituximab } \\
\text { Obinutuzumab }\end{array}$ & CD20 & B cell lymphomas \\
$\begin{array}{l}\text { MOR-208 } \\
\text { Mogamulizumab }\end{array}$ & CD19 & B cell lymphomas \\
$\begin{array}{l}\text { Antibody-drug conjugates } \\
\text { Brentuximab vedotin }\end{array}$ & CCR4 & B cell lymphomas \\
$\begin{array}{l}\text { Polatuzumab vedotin } \\
\text { Denintuzumab mafodotin }\end{array}$ & CD79b cell lymphomas \\
$\begin{array}{l}\text { Coltuximab ravtansine } \\
\text { Loncastuximab tesirine }\end{array}$ & CD19 & Hodgkin lymphoma, ALCL, CTCL \\
\hline $\begin{array}{l}\text { Pinatuzumab vedotin } \\
\text { Inotuzumab ozogamicin }\end{array}$ & CD22 & B cell lymphomas \\
Camidanlumab tesirine & CD22 & B cell lymphomas \\
Bispecific antibodies & B cell and T cell lymphoma, Hodgkin lymphoma \\
Blinatumomab & CD19/CD3 & B cell lymphomas \\
Mosunetuzumab & CD20/CD3 & B cell lymphomas \\
CD20-TCB & CD20/CD3 & B cell lymphomas lymphomas \\
\hline Odronextamab & CD20/CD3 & B cell lymphomas \\
\hline
\end{tabular}

ALCL, anaplastic large-cell lymphoma; CTCL, cutaneous T cell lymphoma.

Other ADCs are in development but have not yet been approved by the FDA. These include ADCs that target CD19 and CD22 on malignant B cells or CD25 on malignant T cells. Several antiCD19 ADCs have been evaluated in B cell lymphomas, including denintuzumab mafodotin (SGN-CD19A), coltuximab ravtansine (SAR3419), and loncastuximab tesirine (ADCT-402) (21-25). Denintuzumab mafodotin, a humanized anti-CD19 antibody conjugated to the drug monomethyl auristatin F (MMAF), and coltuximab ravtansine, an anti-CD19 monoclonal antibody conjugated to the potent cytotoxic maytansinoid drug DM4, both deliver agents that disrupt microtubule assembly. Both agents have moderate clinical activity in patients with relapsed and refractory B cell lymphoma but also resulted in ocular adverse events (26-29). Treatment with loncastuximab tesirine, an anti-CD19 ADC with a pyrrolobenzodiazepine (PBD) dimer payload, has resulted in response rates in relapsed and refractory B cell lymphomas that appear higher than response rates seen with other anti-CD19 ADCs in early-phase trials $(25,30)$, but this will need to be confirmed in subsequent studies. In a similar fashion, two anti-CD22 ADCs have been studied in B cell lymphomas, namely pinatuzumab vedotin, which also has an MMAE payload, and inotuzumab ozogamicin, which has a calicheamicin-based payload. Both agents have been well tolerated, either when administered alone or in combination with rituximab, and both have demonstrated promising response rates (24, 31-34). Similar to loncastuximab tesirine, camidanlumab tesirine (ADCT301) is an anti-CD25 ADC with a PBD dimer payload that delivered promising results in B cell lymphomas and peripheral T cell lymphoma (PTCL) (35). A study of camidanlumab tesirine in relapsed and refractory PTCL and HL is ongoing (NCTO2432235).

An additional way to target the malignant cell and engage the immune system is the use of bispecific T cell engager (BiTE) anti- bodies. These are molecules with dual specificity targeting both a tumor antigen and an immune cell, thereby bringing them in close physical proximity to each other to promote an antitumor immune response. Blinatumomab was the initial BiTE antibody directed against CD19 on B cells and the CD3 $\varepsilon$ subunit of the T cell receptor. Blinatumomab has shown promising efficacy in patients with relapsed and refractory DLBCL but commonly results in substantial neurotoxicity (36). Other T cell-engaging antibodies that target CD20 and CD3 are now also being evaluated in B cell lymphoma. A full-length CD20/CD3 antibody, mosunetuzumab, has been tested in patients with relapsed and refractory B cell lymphoma and was found to be well tolerated with promising response rates, and neurologic toxicities with this agent appeared less common (37). Other CD20/CD3 T cell-engaging antibodies, CD20TCB (RG6026) and odronextamab (REGN1979), have been evaluated in patients with $\mathrm{B}$ cell lymphoma, and responses have been seen in relapsed patients, including patients who failed previous CAR-T cell therapy $(38,39)$.

Targeting $T$ cells and immune checkpoints. While many cells present within the tumor microenvironment in lymphoma are malignant cells, a substantial number of cells present in a malignant lymph node are effector T cells. Most of these cells have been rendered immunologically ineffective and are unable to kill the malignant cells (3). Most of the T cells exhibit features of immune exhaustion, in large part due to signaling through immune checkpoint receptors, particularly the PD- 1 receptor. Therapies that target $\mathrm{T}$ cells and block inhibitory signals through the PD-1 receptor have resulted in dramatic responses in certain lymphoma patients, particularly patients with HL. In other B cell lymphoma subtypes, PD-1 blockade has demonstrated some antitumor activity, but the efficacy has varied substantially by lymphoma subtype (Table 2).

Signaling via the PD-1 receptor is an immune checkpoint that normally serves to dampen immune responses, and tumor cells can co-opt this pathway to evade attack by the host immune system (40). This is particularly true in HL, in which copy number gain or genetic amplification of chromosome 9p24.1 in the malignant Reed-Sternberg cell results in overexpression of PD-L1 on the cell surface (41). Furthermore, incorporation of the Epstein-Barr virus (EBV) into the Reed-Sternberg cell genome activates the JAK/ STAT pathway, resulting in overexpression of PD-L1 on the cells (42). Similarly, subsets of non-Hodgkin lymphomas overexpress PD-L1, and molecular analyses have demonstrated similar genetic alterations involving the PD-1 ligands in certain lymphoma histologies. Overexpression of PD-1 ligands has also been shown to be critical in the pathogenesis of primary mediastinal B cell lymphoma (PMBCL) and EBV-associated post-transplant lymphoproliferative disorders $(41,42)$, and blockade of PD-1/PD-L1 signaling has proven to be highly effective in treating these diseases (43).

In $\mathrm{HL}$, the use of two PD-1-blocking monoclonal antibodies, nivolumab and pembrolizumab, in patients with relapsed and refractory disease has resulted in remarkably high clinical response rates (44-48). Initial phase I clinical trials of these agents suggested that at least two-thirds of patients with HL responded to this treatment $(44,46)$, and this was confirmed in subsequent phase II trials $(45,47,48)$. However, while most patients respond to these treatments, only a minority of patients have a complete response to treatment. More recently, very promising results 
Table 2. Selected trials evaluating PD-1 blockade in relapsed Hodgkin and non-Hodgkin lymphoma

\begin{tabular}{|c|c|c|c|c|c|c|c|c|}
\hline Agent & Phase & Population & $N$ & ORR & CR & DOR (median) & PFS (median) & Ref. \\
\hline Nivolumab & I/II & R/R HL & $\begin{array}{l}\text { Ph. I: } 23 \\
\text { Ph. II: } 243\end{array}$ & $\begin{array}{l}\text { Ph. I: } 87 \% \\
\text { Ph. II: } 69 \%\end{array}$ & $\begin{array}{l}\text { Ph. I: } 17 \% \\
\text { Ph. II: } 16 \%\end{array}$ & $\begin{array}{c}\text { Ph. I: NA } \\
\text { Ph. Il: } 16.6 \text { mo }\end{array}$ & $\begin{array}{c}\text { Ph. I: NA } \\
\text { Ph. II: } 14.7 \text { mo }\end{array}$ & $\begin{array}{c}\text { Ansell et al. } 2015 \text { (44), Armand } \\
\text { et al. } 2018 \text { (45) }\end{array}$ \\
\hline Pembrolizumab & $\mathrm{lb} / \mathrm{II}$ & R/R HL & $\begin{array}{c}\text { Ph. I: } 31 \\
\text { Ph. II: } 210\end{array}$ & $\begin{array}{l}\text { Ph. I: } 65 \% \\
\text { Ph. II: } 69 \%\end{array}$ & $\begin{array}{c}\text { Ph. I: } 16 \% \\
\text { Ph. II: } 22.4 \%\end{array}$ & $\begin{array}{c}\text { Ph. I: NA } \\
\text { Ph. Il: not reached }\end{array}$ & $\begin{array}{l}\text { Ph. I: NA } \\
\text { Ph. II: NA }\end{array}$ & $\begin{array}{c}\text { Armand et al. } 2016 \text { (46), Chen } \\
\text { et al. } 2017 \text { (47) }\end{array}$ \\
\hline Nivolumab & 1 & R/R FL & Ph. I: 10 & Ph. I: $40 \%$ & Ph. I: $10 \%$ & Ph. I: NA & Ph. I: not reached & Lesokhin et al. 2016 (53) \\
\hline Nivolumab & $|/| \mid$ & R/R DLBCL & $\begin{array}{l}\text { Ph. I: } 11 \\
\text { Ph. Il: } 121\end{array}$ & $\begin{array}{c}\text { Ph. I: } 36 \% \\
\text { Ph. II (SCT-failed) 10\% }\end{array}$ & $\begin{array}{l}\text { Ph. I: } 18 \% \\
\text { Ph. II: } 3 \%\end{array}$ & $\begin{array}{c}\text { Ph. I: NA } \\
\text { Ph. Il: } 11 \text { mo }\end{array}$ & $\begin{array}{c}\text { Ph. I: } 7 \text { mo } \\
\text { Ph. Il: } 1.9 \text { mo }\end{array}$ & $\begin{array}{l}\text { Lesokhin et al. } 2016 \text { (53), } \\
\text { Ansell et al. } 2019 \text { (54) }\end{array}$ \\
\hline Pembrolizumab & lb, II & R/R PMBCL & $\begin{array}{l}\text { Ph. Ib: } 21 \\
\text { Ph. II: } 53\end{array}$ & $\begin{array}{l}48 \% \\
45 \%\end{array}$ & $\begin{array}{l}33 \% \\
13 \%\end{array}$ & Not reached & $\begin{array}{c}10.4 \mathrm{mo} \\
5.5 \mathrm{mo}\end{array}$ & Armand et al. 2018 (132) \\
\hline Pembrolizumab & $\|$ & RT & 9 & $44 \%$ & $11 \%$ & NA & $5.4 \mathrm{mo}$ & Ding et al. 2017 (55) \\
\hline Nivolumab & 1 & R/R PTCL & 5 & $40 \%$ & $0 \%$ & NA & $14 \mathrm{mo}$ & Lesokhin et al. 2016 (53) \\
\hline Pembrolizumab & $\|$ & R/R MF/SS & 24 & $38 \%$ & $4 \%$ & NA & Not reached & Khodadoust et al. 2016 (59) \\
\hline Pembrolizumab & II & R/R NKTL & 7 & $100 \%$ & $100 \%$ & $10 \mathrm{mo}$ & Not reached & Kwong et al. 2017 (133) \\
\hline Pembrolizumab & $\|$ & R/R NKTL & 7 & $57 \%$ & $29 \%$ & $4.1 \mathrm{mo}$ & $4.8 \mathrm{mo}$ & Li et al. 2018 (56) \\
\hline
\end{tabular}

CR, complete response; DLBCL, diffuse large B cell lymphoma; DOR, duration of response; FL, follicular lymphoma; HL, Hodgkin lymphoma; MF, mycosis fungoides; NA, not available; NKTL, NK T cell lymphoma; ORR, overall response rate; PFS, progression-free survival; PMBCL, primary mediastinal large B cell lymphoma (ref. 132); R/R, relapsed or refractory; RT, Richter transformation; SCT, stem cell transplant; SS, Sézary syndrome.

with three newer anti-PD-1 antibodies, sintilimab, tislelizumab, and camrelizumab, have been reported in HL patients (49-51). All three of these antibodies have demonstrated very high overall response rates, but, interestingly, more than half the patients treated with these antibodies have had complete responses. While most results have been reported with anti-PD-1 antibodies, promising clinical benefit has also been seen with an antibody directed against PD-L1, avelumab, suggesting that blockade of either the receptor or the ligands inhibits suppressive PD-1 signaling (52).

Although responses to PD-1 blockade have been seen in the majority of HL patients, clinical outcomes with PD-1 blockade in other types of lymphoma have been far less impressive (53-55). Response rates to nivolumab or pembrolizumab in patients with DLBCL or indolent lymphomas have been rather disappointing. The only subgroups of patients with these diseases who had meaningful numbers of clinical responses are those with diseases with amplifications or copy number gain of the PD-L1/2 locus or presence of EBV $(43,56)$. Diseases such as PMBCL, mediastinal gray zone lymphoma, or primary CNS lymphoma frequently have amplifications of chromosome 9p24.1 (43, 57, 58). In these rare entities, responses have been seen in more than a third of patients. In contrast, clinical trials of PD-1 blockade in patients with DLBCL, CLL, multiple myeloma, or follicular lymphoma have found that very few patients respond (53-55). In patients with $\mathrm{T}$ cell lymphomas, a promising number of responses to pembrolizumab have been seen in patients with CTCL (59). However, response rates in PTCL have been modest, and hyperprogression of the disease has been seen in some patients. While amplification of the PD-1 ligands appears to be associated with responses to PD-1-targeting therapies (60), additional biomarkers will need to be developed to identify other lymphoma patients who benefit from this treatment.

Targeting macrophages. The mononuclear phagocyte system is critical in the host's response to pathogens and inflammation. In lymphoma, monocytic cells are central components of the innate immune system, and the prevalence of monocytes in the peripheral blood, as well as that of lymphoma-associated macrophages in malignant lymph nodes, is associated with the prognosis of patients $(61,62)$. Macrophage-mediated phagocytosis is an important mechanism to eliminate diseased and damaged cells, and lymphoma cells, by overexpressing CD47, provide a "don't eat me" signal and thereby avoid eradication. CD47 binds to signal-regulatory protein- $\alpha(\mathrm{SIRP} \alpha)$ to suppress macrophage phagocytosis, and antibodies that block CD47/SIRP $\alpha$ signaling have been developed to promote phagocytosis of lymphoma cells and subsequent increased presentation of tumor antigens to the immune system.

Hu5F9-G4 (5F9) is a first-in-class monoclonal antibody that targets CD47. This antibody has demonstrated promising safety and activity in patients with B cell lymphoma, particularly when administered in combination with rituximab. In an initial clinical trial, this combination resulted in an encouragingly high response rate with complete responses in approximately a quarter of patients. Responses were seen in both follicular lymphoma and DLBCL patients (63). Promising results were also seen with TTI-621 (SIRP $\alpha \mathrm{Fc}$ ), which consists of the CD47 binding domain of human SIRP $\alpha$ linked to the Fc region of human IgG. This molecule is also designed to block CD47 signaling and engage macrophage $\mathrm{Fc} \gamma$ receptors to enhance phagocytosis and promote antitumor activity. Initial results show activity in both B cell and T cell lymphomas, and similarly encouraging results have been seen in combination with rituximab (64).

\section{Small-molecule inhibitors}

While not specifically designed to target cells in the tumor microenvironment, a number of small-molecule inhibitors modulate immune effects in lymphoma patients. It is likely that a number of other chemotherapy treatments also change the composition of the microenvironment or specifically affect subsets of cells within lymph nodes. Two categories of drugs, however, bear special mention. 
Table 3. Summary of CD19 CAR-T therapies that are in late-phase testing or available as standard-of-care practice

\begin{tabular}{|c|c|c|c|}
\hline & ZUMA-1 ${ }^{A}$ & JULIET & TRANSCEND ${ }^{\mathrm{C}}$ \\
\hline Treatment & Axicabtagene ciloleucel & Tisagenlecleucel & Lisocabtagene maraleucel \\
\hline CAR construct & CD19(FMC63)-CD3ל-CD28 & CD19(FMC63)-CD3५-41BB & 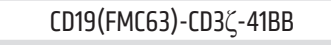 \\
\hline CAR-T composition & Unmanipulated & Unmanipulated & Fixed ratio of CD4 and CD8 T cells \\
\hline Bridging therapy (if needed for disease control) & Steroid only & Allowed & Allowed \\
\hline Median time from enrollment to infusion, days & 17 & 52 & \\
\hline mITT best response ${ }^{0}, \mathrm{ORR} / \mathrm{CR}, \%$ & $82 \% / 54 \%$ & $52 \% / 40 \%$ & $75 \% / 55 \%$ \\
\hline ITT best response $\mathrm{E}^{\mathrm{E}} \mathrm{ORR} / \mathrm{CR}, \%$ & $75 \% / 49 \%$ & $35 \% / 27 \%$ & $64 \% / 43 \%$ \\
\hline 12-Month OS & $59 \%$ & $49 \%$ & $63 \%$ \\
\hline Approved histologies & $\begin{array}{l}\text { DLBCL, high-grade B cell lymphoma, DLBCL } \\
\text { arising from follicular lymphoma, PMBCL }\end{array}$ & $\begin{array}{l}\text { DLBCL, high-grade } B \text { cell lymphoma, } \\
\text { DLBCL arising from follicular lymphoma }\end{array}$ & Not applicable \\
\hline
\end{tabular}

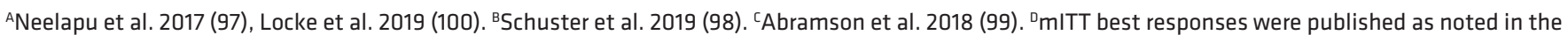
table, based on response rate among patients who were infused. EITT best response represents the calculated response from all patients enrolled in the study. ITT, intent to treat; LD, lymphodepletion; mITT, modified intent to treat; OS, overall survival.

Immunomodulatory drugs. Lenalidomide and other immunomodulatory drugs (IMiDs) have pleotropic effects, including targeting the tumor microenvironment in lymphoma. Lenalidomide has single-agent activity in lymphoma patients, and the combination of lenalidomide plus rituximab $\left(\mathrm{R}^{2}\right)$ has been particularly effective $(65,66)$. This combination produced high response rates that were durable in patients with relapsed and refractory disease (67). In previously untreated patients, the $\mathrm{R}^{2}$ combination has produced results similar to that achieved with chemoimmunotherapy, but potentially with fewer side effects (68). This has led to $\mathrm{R}^{2}$ being considered a standard treatment option for indolent lymphomas.

BTK inhibitors. Ibrutinib, an approved therapy for B cell malignancies, is a covalent inhibitor of Bruton tyrosine kinase (BTK), a member of the B cell receptor (BCR) signaling pathway that is critical for the survival of malignant B cells. Interestingly, this drug also inhibits IL-2-inducible kinase (ITK), an essential enzyme in Th2 T cells. By inhibiting ITK, ibrutinib can shift the balance between Th1 and Th2 T cells and potentially enhance antitumor immune responses (69). It has been shown that the combination of an anti-PD-L1 antibody and ibrutinib suppresses tumor growth in mouse models of lymphoma that are intrinsically insensitive to ibrutinib (70). Current studies are evaluating this combination and also evaluating the use of ibrutinib in combination with cellular therapies.

\section{Vaccines}

Since B cell lymphoma cells express a clonal BCR with an idiotype (Id) in the variable domain, the targeting of Id as a tumor antigen with vaccine approaches has been investigated for indolent lymphomas. Protein vaccines with keyhole limpet hemocyanin (KLH) or with KLH and granulocyte-macrophage colony-stimulating factor (GM-CSF) were tested as adjuvant therapy after chemotherapy or rituximab for follicular lymphoma in three randomized phase III trials (71-73). In general, cellular and humoral anti-Id responses could be identified in some patients, and those with anti-Id responses had prolonged PFS (73). However, the primary endpoints were not met for two of the phase III trials, with no difference in PFS or time to progression between the vaccine and placebo arms $(71,73)$. In a third trial, the disease-free survival was statistically significantly increased for patients in the vaccine arm, although it did not meet the prespecified significance (72). Study design and conduct issues including unbalanced arms for the number of follicular lymphoma patients with poorer prognostic features (71), as well as early stopping of accrual (72), likely contributed to lack of positive findings. Nevertheless, some limitations of the protein vaccine approach were identified, including laborious and costly manufacturing, which can take up to 8 months, and decreased activity in patients with active disease compared with patients in remission. DNA vaccines could be more rapidly manufactured, and this is being examined in clinical testing $(74,75)$.

Cellular vaccines using dendritic cells (DCs) have been studied as a more direct method of priming antigens and eliciting adaptive cellular immunity. Initial trials using DCs pulsed with Id protein demonstrated the ability to induce antilymphoma immunity with a memory response $(76,77)$. To circumvent potential tumor antigen downregulation or escape and to stimulate a broader immune response, DCs have also been loaded with multiple commonly shared tumor antigens, tumor cells, lysates, and RNA (78-80). Modest clinical responses were seen in indolent lymphoma, usually in patients with less bulky disease, as the DC priming is likely still limited by the lymphoma-associated mechanisms of immune suppression $(77,80)$. Combination immunotherapy approaches are now being explored, particularly with intratumor manipulation to generate immunogenic cell death and reset microenvironment immune suppression. Localized tumor treatment such as radiation combined with intratumor loading with DCs or immune agonists such as TLR9 agonists or FLT3L were able to generate abscopal responses, confirming the promise of combination approaches (81-85). 


\section{Adoptive cell therapies}

Given the importance of the effector $\mathrm{T}$ cell response in antitumor immunity with immunotherapy approaches, adoptive transfer of antigen-specific $\mathrm{T}$ cells has been studied. These approaches have focused on expanding $\mathrm{T}$ cells that appear to be tumor specific, or specifically engineering $\mathrm{T}$ cells to bind to the malignant clone.

Non-engineered adoptive cell therapies. EBV-targeted T cells, both autologous and allogeneic, have had the most success as a non-engineered adoptive $\mathrm{T}$ cell therapy to treat EBV-associated lymphoma, including post-transplant lymphoproliferative disorders $(86,87), \mathrm{HL}$, and non-Hodgkin lymphomas such as NK/T cell lymphoma (88-90). To optimize the cytotoxic effect, cytoreduction with chemotherapy remains important to increase the effector/tumor cell ratio and increase effector cell proliferation. Using the same rationale, adoptive transfers of $\mathrm{T}$ cells have also been given post-autologous stem cell transplant $(91,92)$. In addition, NK cells are innate effector cells capable of tumor killing without MHC-mediated antigen presentation. While NK cell manufacturing can be challenging, recent advances have enabled phase I/II studies using autologous cells $(93,94)$, haploidentical cells (95), or the NK cell line NK-92 (96) to treat B cell lymphoma. Increased serum levels of IL-15, a cytokine that promotes proliferation, were associated with clinical response (95), indicating a role for combining this cytokine with immune cell therapy to promote its efficacy.

CAR-T cell therapy. CAR-T cell therapy has garnered the most excitement as the first FDA-approved cellular therapy in lymphoma. In this individualized therapy, the patient's own T cells are genetically transduced with lentiviral or retroviral vectors ex vivo to express a CAR that enables the CAR-positive $\mathrm{T}$ cells to bypass MHC presentation for recognition of antigens and activation of $\mathrm{T}$ cells with fused intracellular $\mathrm{CD} 3 \zeta$ and costimulatory signaling domains. Two CD19-targeting CAR-T approaches, axicabtagene ciloleucel (Axi-cel) and tisagenlecleucel, are approved to treat relapsed, refractory aggressive B cell non-Hodgkin lymphoma, specifically high-grade B cell lymphoma, DLBCL, transformed follicular lymphoma, and PMBCL (Axi-cel only) (97, 98). A third CD19 CAR-T therapy, lisocabtagene maraleucel (liso-cel), is anticipated to be reviewed by the FDA in early 2020 (99). All three CAR-Ts use the same single-chain variable fragment domain $(\mathrm{scFv})$ in the extracellular domain to recognize CD19, and differ in the intracellular cosignaling domain, with CD28 for Axi-cel and 41BB for tisagenlecleucel and liso-cel. Liso-cel is also administered in a fixed 1:1 ratio of CD4 and CD8 CAR-T cells. Patients receive lymphodepletion chemotherapy, most commonly with cyclophosphamide and fludarabine, or bendamustine (tisagenlecleucel), before infusion of CAR-T cells. Results from the three pivotal trials for these CAR-T therapies showed impressive response rate and durable responses in a subset of patients, including ongoing complete remissions with up to 2 years of follow-up $(98,100)$. The response rate was not affected by poor prognostic factors including age, International Prognostic Index (IPI) score, cell of origin, and refractory status $(97,98)$. While longer follow-up is needed to understand whether there are differences in the durability of response and survival across the three CD19 CAR-T therapies, the rate of leukapheresed patients receiving CAR-T dosing, which can be affected by manufacturing logistics and the aggres- siveness of the patient's disease during the manufacturing time, is a feasibility issue and impacts the intent-to-treat response rates among the three CAR-T therapies (Table 3). Since its FDA approval, real-world practice outcomes have been reported for Axi-cel $(101,102)$. Even though approximately half of the treated patients did not meet pivotal trial eligibility criteria for performance status or organ function, the manufacturing success rate and the response rate remain comparable to the trial results.

Cytokine release syndrome (CRS) and neurotoxicities (immune cell-associated neurologic syndromes, or ICANS) most commonly present in the first month after infusion. With current severitybased management approaches that use cytokine blockade and steroids, CRS and ICANS are usually completely reversible and only rarely fatal $(97,98,103-106)$. However, the specialized expertise required to manage these complications has limited the administration of CAR-T therapies to tertiary centers. Multiple investigations are planned to examine alternative management for CRS and ICANS (107-109). Long-term toxicities include B cell aplasia and associated hypogammaglobulinemia. Unlike B cell acute lymphoblastic leukemia, B cell and immunoglobulin recovery can occur in $\mathrm{B}$ cell lymphoma patients and is not associated with increased risk of relapse $(98,100)$. Secondary malignancies remain a potential risk with this gene therapy, although longer follow-up is needed to understand the true risk.

Ongoing investigations of CD19-targeted CAR-T include its use in earlier lines of therapy: in a second-line setting in a randomized control study comparing with autologous stem cell transplant (NCT03391466, NCT03575351, NCT03570892) and in a first- and second-line setting for patients with high-risk disease or patients who are not eligible for stem cell transplant (NCT03761056, NCT03483103). The use of CAR-T cell therapy is being tested in other B cell non-Hodgkin lymphomas, including mantle cell lymphoma, follicular lymphoma, and marginal zone lymphoma.

Multiple strategies are also being investigated to improve response rates in CAR-T therapy. Lymphodepletion chemotherapy was recognized early on as an important contributor to CAR-T expansion, persistence, and associated response (110, 111); however, it also contributes to prolonged cytopenia and infection and likely to secondary malignancy. Alternative immune-modulating methods such as radiation are being explored as lymphodepletion approaches (112). The intrinsic functional capacity and immunosuppressive signaling of CAR-T have been found to be associated with decreased response (113). CAR engineering with additional costimulatory signaling, increased paracrine signaling by stimulatory cytokines such as IL-15 and IL-12, or editing to remove inhibitory signaling is in development (114-116). Combination therapies with checkpoint blockade (NCT03310619, NCT03630159, NCT02926833) and immune modulators such as ibrutinib, 41BB agonist, and CC-122 (NCT03310619, NCT03331198, NCT03704298) are just a few of the strategies being investigated. Tumor antigen escape has also been implicated in lymphoma relapse $(97,117-119)$. To overcome this, alternative antigen and dual antigen targeting with CD20 and CD22 CAR-T have shown promising responses (120-124).

Clinical responses to the CAR-T approach beyond $\mathrm{B}$ cell non-Hodgkin lymphoma have not been as impressive to date. 
CD30 CAR-T cells have been tested in HL and T cell lymphoma $(125,126)$, but responses may have been restricted by the immunosuppressive microenvironment that limits CAR-T trafficking and activation $(125,127)$. CD5 and CD7 CAR-T cells have been tested in T cell lymphoma $(128,129)$. Interestingly, while both CD5 and CD7 are expressed on normal T cells, fratricide from CD5 CAR-T is only seen transiently, while malignant T cells were effectively killed in vitro and in vivo $(128,129)$. The disparate killing effect was found to be due to differential sensitivities to granzyme or Fas-mediated killing between malignant and nonmalignant T cells $(128,129)$. Early results of a phase I clinical trial suggest that CD5 CAR-T cells also do not induce complete T cell aplasia, although completion of the study is needed to fully define the efficacy and toxicity profile (NCT03081910). For CD7targeting CAR-T cells, gene editing approaches such as CRISPR to block endogenous CD7 expression were effective at preventing fratricide while maintaining cytotoxicity against malignant cells in a preclinical model $(128,129)$. Several studies have begun enrolling patients and are testing CD7 CAR-Ts (NCT03690011, NCT04033302, NCT04004637). Alternatively, TCRB1-targeting CAR-T is being tested in clinic to target $\mathrm{T}$ cell lymphoma that only expresses TCRB1 in the malignant clone (130).

To overcome the intrinsic defective capacity of autologous $\mathrm{T}$ cells in lymphoma patients, allogeneic CAR-T could be generated from a healthier $\mathrm{T}$ cell, could be more readily available offthe-shelf, and could thereby bypass the manufacturing wait time. Early results for allo-CAR-T in B cell malignancies are encouraging (131). Finally, CAR-NK cells are also being tested, as NK cells may have innate antitumor immunity capacity beyond the CAR-induced activities. Umbilical cord blood, haploidentical allogeneic donors, and the NK cell line NK-92 are being explored as potential sources for therapeutic development (NCT02742727, NCT02892695, NCT03056339, NCT03579927).

\section{Conclusion}

Since the introduction of rituximab, immunotherapy has been central to the treatment of lymphoma. The armamentarium of novel immunotherapies continues to expand, with the introduction of newer monoclonal antibodies that are engineered to enhance tumor cell killing or that target different cellular antigens; effective new ADCs; optimized CAR-T cells; and antibodies that block new immune checkpoints. The ongoing development of new and effective immunotherapies presents both important therapeutic opportunities and new challenges regarding how to introduce these drugs into the clinic. Many unanswered questions remain, including how to sequence these novel immunotherapies, how to combine them with cellular immunotherapies, and when to administer them in the course of a patient's therapy. Finally, the reality is that despite the promising antitumor activity exhibited by many novel immunotherapies, there are many patients who do not respond to these agents. It will therefore be important to identify predictive biomarkers for immunotherapeutic agents and combinations, so as to treat the subset of patients most likely to benefit from these approaches and avoid toxicity and cost in the remaining patients. With a rapidly expanding range of effective immunotherapy options for the treatment of lymphoma, optimizing immunotherapy for lymphoma patients will remain a critically important task.

Address correspondence to: Stephen M. Ansell, Division of Hematology, Mayo Clinic, 200 First Street, Rochester, Minnesota 55905, USA. Phone: 507.266.2040; Email: Ansell.Stephen@mayo.edu.
1. Ansell SM. Non-Hodgkin lymphoma: diagnosis and treatment. Mayo Clin Proc. 2015;90(8):1152-1163.

2. Scott DW, Gascoyne RD. The tumour microenvironment in B cell lymphomas. Nat Rev Cancer. 2014;14(8):517-534.

3. Yang ZZ, Liang AB, Ansell SM. T-cell-mediated antitumor immunity in B-cell non-Hodgkin lymphoma: activation, suppression and exhaustion. Leuk Lymphoma . 2015;56(9):2498-2504.

4. McLaughlin P, et al. Rituximab chimeric antiCD20 monoclonal antibody therapy for relapsed indolent lymphoma: half of patients respond to a four-dose treatment program. J Clin Oncol. 1998;16(8):2825-2833.

5. Colombat P, et al. Rituximab (anti-CD20 monoclonal antibody) as single first-line therapy for patients with follicular lymphoma with a low tumor burden: clinical and molecular evaluation. Blood. 2001;97(1):101-106.

6. Hiddemann W, et al. Frontline therapy with rituximab added to the combination of cyclophosphamide, doxorubicin, vincristine, and prednisone (CHOP) significantly improves the outcome for patients with advanced-stage follicular lymphoma compared with therapy with CHOP alone: results of a prospective randomized study of the German Low-Grade Lymphoma Study Group. Blood. 2005;106(12):3725-3732.
7. Coiffier B, et al. CHOP chemotherapy plus rituximab compared with CHOP alone in elderly patients with diffuse large-B-cell lymphoma. N Engl J Med. 2002;346(4):235-242.

8. Czuczman MS, et al. Phase II trial of galiximab (anti-CD80 monoclonal antibody) plus rituximab (CALGB 50402): Follicular Lymphoma International Prognostic Index (FLIPI) score is predictive of upfront immunotherapy responsiveness. Ann Oncol. 2012;23(9):2356-2362.

9. Grant BW, et al. A phase 2 trial of extended induction epratuzumab and rituximab for previously untreated follicular lymphoma: CALGB 50701. Cancer. 2013;119(21):3797-3804.

10. Goede V, et al. Obinutuzumab plus chlorambucil in patients with CLL and coexisting conditions. NEngl JMed. 2014;370(12):1101-1110.

11. Marcus R, et al. Obinutuzumab for the first-line treatment of follicular lymphoma. $N$ Engl J Med. 2017;377(14):1331-1344.

12. Cheson BD, et al. Overall survival benefit in patients with rituximab-refractory indolent non-Hodgkin lymphoma who received obinutuzumab plus bendamustine induction and obinutuzumab maintenance in the GADOLIN study. J Clin Oncol. 2018;36(22):2259-2266.

13. Vitolo U, et al. Obinutuzumab or rituximab plus cyclophosphamide, doxorubicin, vincristine, and prednisone in previously untreated diffuse large B-cell lymphoma. J Clin Oncol. 2017;35(31):3529-3537.

14. Salles G, et al. Primary analysis results of the single-arm phase II study of MOR208 plus lenalidomide in patients with relapsed or refractory diffuse large B-cell lymphoma (L-MIND). Hematol Oncol. 2019;37(S2):173-174.

15. Ishida $\mathrm{T}$, et al. Defucosylated anti-CCR4 monoclonal antibody (KW-0761) for relapsed adult T-cell leukemia-lymphoma: a multicenter phase II study. JClin Oncol. 2012;30(8):837-842.

16. Ogura M, et al. Multicenter phase II study of mogamulizumab (KW-0761), a defucosylated anti-cc chemokine receptor 4 antibody, in patients with relapsed peripheral T-cell lymphoma and cutaneous T-cell lymphoma. J Clin Oncol. 2014;32(11):1157-1163.

17. Kim YH, et al. Mogamulizumab versus vorinostat in previously treated cutaneous T-cell lymphoma (MAVORIC): an international, open-label, randomised, controlled phase 3 trial. Lancet Oncol. 2018;19(9):1192-1204.

18. Younes A, et al. Results of a pivotal phase II study of brentuximab vedotin for patients with relapsed or refractory Hodgkin's lymphoma. JClin Oncol. 2012;30(18):2183-2189.

19. Connors JM, et al. Brentuximab vedotin with chemotherapy for stage III or IV Hodgkin's lymphoma. NEngl J Med. 2018;378(4):331-344. 
20. Younes A, et al. Brentuximab vedotin (SGN-35) for relapsed CD30-positive lymphomas. $\mathrm{N} \mathrm{Engl} \mathrm{J}$ Med. 2010;363(19):1812-1821.

21. Pro B, et al. Brentuximab vedotin (SGN-35) in patients with relapsed or refractory systemic anaplastic large-cell lymphoma: results of a phase II study. JClin Oncol. 2012;30(18):2190-2196.

22. Prince HM, et al. Brentuximab vedotin or physician's choice in CD30-positive cutaneous T-cell lymphoma (ALCANZA): an international, open-label, randomised, phase 3, multicentre trial. Lancet. 2017;390(10094):555-566.

23. Horwitz S, et al. Brentuximab vedotin with chemotherapy for CD30-positive peripheral T-cell lymphoma (ECHELON-2): a global, double-blind, randomised, phase 3 trial. Lancet . 2019;393(10168):229-240

24. [No authors listed]. Preliminary results of a phase II randomized study (ROMULUS) of polatuzumab vedotin or pinatuzumab vedotin plus rituximab in patients with relapsed/refractory non-Hodgkin lymphoma (NHL). Clin Adv Hematol Oncol. 2014;12(8 suppl 16):15-18.

25. Sehn LH, Kamdar M, Herrera AF. Randomized phase 2 trial of polatuzumab vedotin (pola) with bendamustine and rituximab (BR) in relapsed/ refractory (r/r) FL and DLBCL. JClin Oncol. 2018;36(15 suppl):7507.

26. Moskowitz $\mathrm{CH}$, et al. A Phase 1 study of denintuzumab mafodotin (SGN-CD19A) in relapsed/ refractory B-lineage non-Hodgkin lymphoma. Blood. 2015;126:182.

27. Ribrag V, et al. A dose-escalation study of SAR3419, an anti-CD19 antibody maytansinoid conjugate, administered by intravenous infusion once weekly in patients with relapsed/refractory B-cell non-Hodgkin lymphoma. Clin Cancer Res. 2014;20(1):213-220.

28. Coiffier B, et al. A phase II, single-arm, multicentre study of coltuximab ravtansine (SAR3419) and rituximab in patients with relapsed or refractory diffuse large B-cell lymphoma. Br J Haematol. 2016;173(5):722-730.

29. Trnĕný M, et al. A phase II multicenter study of the anti-CD19 antibody drug conjugate coltuximab ravtansine (SAR3419) in patients with relapsed or refractory diffuse large B-cell lymphoma previously treated with rituximabbased immunotherapy. Haematologica. 2018;103(8):1351-1358.

30. Kahl BS, et al. Encouraging early results from the first in-human clinical trial of ADCT- 402 (loncastuximab tesirine), a novel pyrrolobenzodiazepine-based antibody drug conjugate, in relapsed/refractory B-cell lineage non-Hodgkin lymphoma. Blood. 2017;130(suppl 1):187.

31. Fayad L, et al. Safety and clinical activity of a combination therapy comprising two antibody-based targeting agents for the treatment of non-Hodgkin lymphoma: results of a phase I/ II study evaluating the immunoconjugate inotuzumab ozogamicin with rituximab. JClin Oncol. 2013;31(5):573-583.

32. Ogura M, et al. Phase I study of anti-CD22 immunoconjugate inotuzumab ozogamicin plus rituximab in relapsed/refractory B-cell non-Hodgkin lymphoma. Cancer Sci. 2012;103(5):933-938.

33. Wagner-Johnston ND, et al. A phase 2 study of inotuzumab ozogamicin and rituximab, followed by autologous stem cell transplant in patients with relapsed/refractory diffuse large B-cell lymphoma. Leuk Lymphoma . 2015;56(10):2863-2869.

34. Dang NH, et al. Randomized, phase 3 trial of inotuzumab ozogamicin plus rituximab versus chemotherapy plus rituximab for relapsed/refractory aggressive B-cell non-Hodgkin lymphoma. $\mathrm{Br} \mathrm{J}$ Haematol. 2018;182(4):583-586.

35. Collins GP, et al. ADCT-301 (camidanlumab tesirine), a novel pyrrolobenzodiazepine-based CD25-targeting antibody drug conjugate, in a phase 1 study of relapsed/refractory non-Hodgkin lymphoma shows activity in T-cell lymphoma. ADC Therapeutics. https://adctherapeutics.com/ adctsite/wp-content/uploads/ASH 301 2018. pdf. Accessed January 8, 2020

36. Viardot A, et al. Phase 2 study of the bispecific $\mathrm{T}$-cell engager (BiTE) antibody blinatumomab in relapsed/refractory diffuse large B-cell lymphoma. Blood. 2016;127(11):1410-1416.

37. Budde LE, et al. Mosunetuzumab, a full-length bispecific CD20/CD3 antibody, displays clinical activity in relapsed/refractory B-cell non-Hodgkin lymphoma (NHL): interim safety and efficacy results from a phase 1 study. Blood. 2018;132(suppl 1):399.

38. Hutchings M, et al. CD20-TCB (RG6026), a novel " $2: 1$ " format $\mathrm{T}$-cell-engaging bispecific antibody, induces complete remissions in relapsed/refractory B-cell non-Hodgkin's lymphoma: preliminary results from a phase I first in human trial. Blood. 2018;132(suppl 1):8618783.

39. Bannerji R, et al. Emerging clinical activity of REGN1979, an anti-CD20 $\mathrm{x}$ anti-CD3 bispecific antibody, in patients with relapsed/refractory follicular lymphoma (FL), diffuse large B-cell lymphoma (DLBCL), and other B-cell non-Hodgkin lymphoma (B-NHL) subtypes. Regeneron. https://investor.regeneron.com/staticfiles/86ef5b93-c6f3-47f0-8be8-43394dd709fa. Accessed January 8, 2020.

40. Pardoll DM. The blockade of immune checkpoints in cancer immunotherapy. Nat Rev Cancer. 2012;12(4):252-264.

41. Green MR, et al. Integrative analysis reveals selective 9p24.1 amplification, increased PD-1 ligand expression, and further induction via JAK2 in nodular sclerosing Hodgkin lymphoma and primary mediastinal large B-cell lymphoma. Blood. 2010;116(17):3268-3277.

42. Green MR, et al. Constitutive AP-1 activity and EBV infection induce PD-L1 in Hodgkin lymphomas and posttransplant lymphoproliferative disorders: implications for targeted therapy. Clin Cancer Res. 2012;18(6):1611-1618.

43. Zinzani PL, et al. Safety and tolerability of pembrolizumab in patients with relapsed/refractory primary mediastinal large B-cell lymphoma. Blood. 2017;130(3):267-270.

44. Ansell SM, et al. PD-1 blockade with nivolumab in relapsed or refractory Hodgkin's lymphoma. N Engl J Med. 2015;372(4):311-319.

45. Armand P, et al. Nivolumab for relapsed/refractory classic Hodgkin lymphoma after failure of autologous hematopoietic cell transplantation: extended follow-up of the multicohort singlearm phase II CheckMate 205 Trial. JClin Oncol.
2018;36(14):1428-1439.

46. Armand P, et al. Programmed death-1 blockade with pembrolizumab in patients with classical Hodgkin lymphoma after brentuximab vedotin failure. JClin Oncol. 2016;34(31):3733-3739.

47. Chen R, et al. Phase II study of the efficacy and safety of pembrolizumab for relapsed/refractory classic Hodgkin lymphoma. J Clin Oncol. 2017;35(19):2125-2132.

48. Younes A, et al. Nivolumab for classical Hodgkin's lymphoma after failure of both autologous stemcell transplantation and brentuximab vedotin: a multicentre, multicohort, single-arm phase 2 trial. Lancet Oncol. 2016;17(9):1283-1294.

49. Shi Y, et al. Safety and activity of sintilimab in patients with relapsed or refractory classical Hodgkin lymphoma (ORIENT-1): a multicentre, single-arm, phase 2 trial. Lancet Haematol. 2019;6(1):e12-e19.

50. Song Y, et al. Tislelizumab (BGB-A317) for relapsed/refractory classical Hodgkin lymphoma: preliminary efficacy and safety results from a phase 2 study. Blood. 2018;132(suppl_1):682.

51. Song Y, et al. A single-arm, multicenter, phase II study of camrelizumab in relapsed or refractory classical Hodgkin lymphoma. Clin Cancer Res. 2019;25(24):7363-7369.

52. Chen R, et al. Blockade of the PD-1 checkpoint with anti-PD-L1 antibody avelumab is sufficient for clinical activity in relapsed/refractory classical Hodgkin lymphoma (CHL). Hematol Oncol. 2017;35(suppl 2):67.

53. Lesokhin AM, et al. Nivolumab in patients with relapsed or refractory hematologic malignancy: preliminary results of a phase Ib study. J Clin Oncol. 2016;34(23):2698-2704.

54. Ansell SM, et al. Nivolumab for relapsed/refractory diffuse large B-cell lymphoma in patients ineligible for or having failed autologous transplantation: a single-arm, phase II study. J Clin Oncol. 2019;37(6):481-489.

55. Ding W, et al. Pembrolizumab in patients with CLL and Richter transformation or with relapsed CLL. Blood. 2017;129(26):3419-3427.

56. Li X, et al. Activity of pembrolizumab in relapsed/ refractory NK/T-cell lymphoma. J Hematol Oncol. 2018;11(1):15.

57. Melani C, et al. PD-1 blockade in mediastinal gray-zone lymphoma. $N$ Engl J Med. 2017;377(1):89-91.

58. Nayak L, et al. PD-1 blockade with nivolumab in relapsed/refractory primary central nervous system and testicular lymphoma. Blood. 2017;129(23):3071-3073

59. Khodadoust MS, et al. Pembrolizumab in relapsed and refractory mycosis fungoides and Sézary syndrome: a multicenter phase II study. JClin Oncol. 2020;38(1):20-28.

60. Roemer MGM, et al. Major histocompatibility complex class II and programmed death ligand 1 expression predict outcome after programmed death 1 blockade in classic Hodgkin lymphoma. JClin Oncol. 2018;36(10):942-950.

61. Kridel R, et al. The prognostic impact of CD163-positive macrophages in follicular lymphoma: a study from the BC Cancer Agency and the Lymphoma Study Association. Clin Cancer Res. 2015;21(15):3428-3435. 
62. Wilcox RA, et al. The absolute monocyte count is associated with overall survival in patients newly diagnosed with follicular lymphoma. Leuk Lymphoma. 2012;53(4):575-580.

63. Advani R, et al. CD47 blockade by Hu5F9-G4 and rituximab in non-Hodgkin's lymphoma. N Engl J Med. 2018;379(18):1711-1721.

64. Johnson LDS, et al. Targeting CD47 in Sézary syndrome with SIRP $\alpha$ Fc. Blood $A d v$. 2019;3(7):1145-1153.

65. Leonard JP, et al. Randomized trial of lenalidomide alone versus lenalidomide plus rituximab in patients with recurrent follicular lymphoma: CALGB 50401 (Alliance). JClin Oncol. 2015;33(31):3635-3640

66. Martin P, et al. A phase II trial of lenalidomide plus rituximab in previously untreated follicular non-Hodgkin's lymphoma (NHL): CALGB 50803 (Alliance). Ann Oncol. 2017;28(11):2806-2812.

67. Izutsu K, et al. Analysis of Japanese patients from the AUGMENT phase III study of lenalidomide + rituximab $\left(\mathrm{R}^{2}\right)$ vs. rituximab + placebo in relapsed/refractory indolent non-Hodgkin lymphoma [published online ahead of print December 19, 2019]. Int J Hematol. https://doi. org/10.1007/s12185-019-02802-y.

68. Morschhauser F, et al. Rituximab plus lenalidomide in advanced untreated follicular lymphoma. N Engl JMed. 2018;379(10):934-947.

69. Dubovsky JA, et al. Ibrutinib is an irreversible molecular inhibitor of ITK driving a Th1selective pressure in T lymphocytes. Blood. 2013;122(15):2539-2549.

70. Sagiv-Barfi I, Kohrt HE, Czerwinski DK, Ng PP, Chang BY, Levy R. Therapeutic antitumor immunity by checkpoint blockade is enhanced by ibrutinib, an inhibitor of both BTK and ITK. Proc Natl Acad Sci U S A . 2015;112(9):E966-E972.

71. Freedman A, et al. Placebo-controlled phase III trial of patient-specific immunotherapy with mitumprotimut-T and granulocyte-macrophage colony-stimulating factor after rituximab in patients with follicular lymphoma. J Clin Oncol. 2009;27(18):3036-3043.

72. Schuster SJ, et al. Vaccination with patient-specific tumor-derived antigen in first remission improves disease-free survival in follicular lymphoma. JClin Oncol. 2011;29(20):2787-2794.

73. Levy R, et al. Active idiotypic vaccination versus control immunotherapy for follicular lymphoma. J Clin Oncol. 2014;32(17):1797-1803.

74. Meleshko AN, Petrovskaya NA, Savelyeva N, Vashkevich KP, Doronina SN, Sachivko NV. Phase I clinical trial of idiotypic DNA vaccine administered as a complex with polyethylenimine to patients with B-cell lymphoma. Hum Vaccin Immunother. 2017;13(6):1-6.

75. Thomas SK, et al. Phase I study of an active immunotherapy for asymptomatic phase Lymphoplasmacytic lymphoma with DNA vaccines encoding antigen-chemokine fusion: study protocol. BMC Cancer. 2018;18(1):187.

76. Hsu FJ, et al. Vaccination of patients with B-cell lymphoma using autologous antigen-pulsed dendritic cells. Nat Med.1996;2(1):52-58.

77. Timmerman JM, et al. Idiotype-pulsed dendritic cell vaccination for B-cell lymphoma: clinical and immune responses in 35 patients. Blood.
2002;99(5):1517-1526.

78. Chen W, et al. Efficient induction of antitumo $\mathrm{T}$ cell immunity by exosomes derived from heat-shocked lymphoma cells. Eur J Immunol. 2006;36(6):1598-1607.

79. Ni X, et al. Induction of T-cell responses against cutaneous T-cell lymphomas ex vivo by autologous dendritic cells transfected with amplified tumor mRNA. J Invest Dermatol. 2008;128(11):2631-2639.

80. Di Nicola M, et al. Vaccination with autologous tumor-loaded dendritic cells induces clinical and immunologic responses in indolent B-cell lymphoma patients with relapsed and measurable disease: a pilot study. Blood. 2009;113(1):18-27.

81. Betting DJ, Yamada RE, Kafi K, Said J, van Rooijen N, Timmerman JM. Intratumoral but not systemic delivery of $\mathrm{CpG}$ oligodeoxynucleotide augments the efficacy of anti-CD20 monoclonal antibody therapy against B cell lymphoma. JImmunother. 2009;32(6):622-631.

82. Brody JD, et al. In situ vaccination with a TLR9 agonist induces systemic lymphoma regression: a phase I/II study. J Clin Oncol. 2010;28(28):4324-4332.

83. Kim $\mathrm{YH}$, et al. In situ vaccination against mycosis fungoides by intratumoral injection of a TLR9 agonist combined with radiation: a phase $1 / 2$ study. Blood. 2012;119(2):355-363.

84. Kolstad A, et al. Sequential intranodal immunotherapy induces antitumor immunity and correlated regression of disseminated follicular lymphoma. Blood. 2015;125(1):82-89.

85. Hammerich L, et al. Systemic clinical tumor regressions and potentiation of $\mathrm{PD} 1$ blockade with in situ vaccination. Nat Med 2019;25(5):814-824.

86. Rooney CM, et al. Infusion of cytotoxic T cells for the prevention and treatment of Epstein-Barr virus-induced lymphoma in allogeneic transplant recipients. Blood. 1998;92(5):1549-1555.

87. Heslop HE, et al. Long-term outcome of EBVspecific T-cell infusions to prevent or treat EBVrelated lymphoproliferative disease in transplant recipients. Blood. 2010;115(5):925-935.

88. Chiou FK, Beath SV, Wilkie GM, Vickers MA, Morland B, Gupte GL. Cytotoxic T-lymphocyte therapy for post-transplant lymphoproliferative disorder after solid organ transplantation in children. Pediatr Transplant. 2018;22(2):e13133.

89. Bollard CM, et al. Sustained complete responses in patients with lymphoma receiving autologous cytotoxic T lymphocytes targeting Epstein-Barr virus latent membrane proteins. JClin Oncol. 2014;32(8):798-808.

90. Gallot G, et al. T-cell therapy using a bank of EBV-specific cytotoxic $T$ cells: lessons from a phase I/II feasibility and safety study. J Immunother. 2014;37(3):170-179.

91. Bollard CM, et al. Complete responses of relapsed lymphoma following genetic modification of tumor-antigen presenting cells and T-lymphocyte transfer. Blood. 2007;110(8):2838-2845

92. Cho SG, et al. Long-term outcome of extranodal NK/T cell lymphoma patients treated with postremission therapy using EBV LMP1 and LMP2a-specific CTLs. Mol Ther. 2015;23(8):1401-1409.
93. Koehl U, et al. Advances in clinical NK cell studies: donor selection, manufacturing and quality control. Oncoimmunology. 2016;5(4):e1115178.

94. Tanaka J, et al. Phase I study of cellular therapy using ex vivo expanded NK cell from autologous peripheral blood mononuclear cells combined with rituximab-containing chemotherapy for relapsed CD20-positive malignant lymphoma patients [published online ahead of print August 8, 2019]. Haematologica. https://doi. org/10.3324/haematol.2019.226696.

95. Bachanova V, et al. Haploidentical natural killer cells induce remissions in non-Hodgkin lymphoma patients with low levels of immunesuppressor cells. Cancer Immunol Immunother. 2018;67(3):483-494.

96. Williams BA, et al. A phase I trial of NK-92 cells for refractory hematological malignancies relapsing after autologous hematopoietic cell transplantation shows safety and evidence of efficacy. Oncotarget. 2017;8(51):89256-89268.

97. Neelapu SS, et al. Axicabtagene ciloleucel CAR T-cell therapy in refractory large B-cell lymphoma. N Engl J Med. 2017;377(26):2531-2544.

98. Schuster SJ, et al. Tisagenlecleucel in adult relapsed or refractory diffuse large B-cell lymphoma. NEngl J Med. 2019;380(1):45-56.

99. Abramson JS, et al. Updated safety and long term clinical outcomes in TRANSCEND NHL 001, pivotal trial of lisocabtagene maraleucel (JCAR017) in R/R aggressive NHL. J Clin Oncol. 2018;36(suppl 15):7505

100.Locke FL, et al. Long-term safety and activity of axicabtagene ciloleucel in refractory large B-cel lymphoma (ZUMA-1): a single-arm, multicentre, phase 1-2 trial. Lancet Oncol. 2019;20(1):31-42.

101. Jacobson CA, et al. Axicabtagene ciloleucel in the real world: outcomes and predictors of response, resistance and toxicity. Blood. 2018;132(suppl 1):92.

102. Nastoupil LJ, et al. Axicabtagene ciloleucel (axi-cel) CD19 chimeric antigen receptor (CAR) $\mathrm{T}$-cell therapy for relapsed/refractory large B-cell lymphoma: real world experience. Blood. 2018;132(suppl 1):91.

103. Lee DW, et al. Current concepts in the diagnosis and management of cytokine release syndrome. Blood. 2014;124(2):188-195.

104. Neelapu SS, et al. Chimeric antigen receptor T-cell therapy - assessment and management of toxicities. Nat Rev Clin Oncol. 2018;15(1):47-62.

105. Brudno JN, Kochenderfer JN. Recent advances in CAR T-cell toxicity: mechanisms, manifestations and management. Blood Rev. 2019;34:45-55.

106. Locke FL, et al. Phase 1 results of ZUMA-1: a multicenter study of KTE-C19 anti-CD19 CAR T cell therapy in refractory aggressive lymphoma. $\mathrm{Mol}$ Ther. 2017;25(1):285-295.

107. Sterner RM, Cox MJ, Sakemura R, Kenderian SS. Using CRISPR/Cas9 to knock out GM-CSF in CAR-T cells. J Vis Exp. 2019;(149):e59629.

108. Sterner RM, et al. GM-CSF inhibition reduces cytokine release syndrome and neuroinflammation but enhances CAR-T cell function in xenografts. Blood. 2019;133(7):697-709.

109. Eyquem J, et al. Targeting a CAR to the TRAC locus with CRISPR/Cas9 enhances tumour rejection. Nature. 2017;543(7643):113-117. 
110. Turtle CJ, et al. CD19 CAR-T cells of defined $\mathrm{CD}^{+}: \mathrm{CD}^{+}$composition in adult B cell ALL patients. J Clin Invest. 2016;126(6):2123-2138.

111. Turtle CJ, et al. Immunotherapy of non-Hodgkin's lymphoma with a defined ratio of $\mathrm{CD} 8+$ and CD4+ CD19-specific chimeric antigen receptor-modified T cells. Sci Transl Med. 2016;8(355):355ra116.

112. Sim AJ, et al. Radiation therapy as a bridging strategy for CAR T cell therapy with axicabtagene ciloleucel in diffuse large B-cell lymphoma. Int $J$ Radiat Oncol Biol Phys. 2019;105(5):1012-1021.

113. Fraietta JA, et al. Determinants of response and resistance to $\mathrm{CD} 19$ chimeric antigen receptor (CAR) T cell therapy of chronic lymphocytic leukemia. Nat Med. 2018;24(5):563-571.

114. Avanzi MP, et al. Engineered tumor-targeted T cells mediate enhanced anti-tumor efficacy both directly and through activation of the endogenous immune system. Cell Rep. 2018;23(7):2130-2141.

115. Yeku OO, Purdon TJ, Koneru M, Spriggs D, Brentjens RJ. Armored CAR T cells enhance antitumor efficacy and overcome the tumor microenvironment. Sci Rep. 2017;7(1):10541.

116. Rafiq S, et al. Targeted delivery of a PD-1-blocking scFv by CAR-T cells enhances anti-tumor efficacy in vivo. Nat Biotechnol. 2018;36(9):847-856.

117. Bukhari A, et al. Rapid relapse of large B-cell lymphoma after CD19 directed CAR-T-cell therapy due to CD-19 antigen loss. Am J Hematol. 2019;94(10):E273-E275.

118. Schuster SJ, et al. Chimeric antigen receptor $\mathrm{T}$ cells in refractory B-cell lymphomas. $N$ Engl J Med. 2017;377(26):2545-2554.
119. Shalabi H, et al. Sequential loss of tumor surface antigens following chimeric antigen receptor $\mathrm{T}$-cell therapies in diffuse large B-cell lymphoma. Haematologica. 2018;103(5):e215-e218.

120. Hossain N, et al. Phase I experience with a bi-specific CAR targeting CD19 and CD22 in adults with B-cell malignancies. Blood. 2018;132(suppl 1):490.

121. Shah NN, et al. Results of a phase I study of bispecific anti-CD19, anti-CD20 chimeric antigen receptor (CAR) modified $\mathrm{T}$ cells for relapsed, refractory, non-Hodgkin lymphoma. J Clin Oncol. 2019;37(suppl 15):2510.

122. Till BG, et al. CD20-specific adoptive immunotherapy for lymphoma using a chimeric antigen receptor with both $\mathrm{CD} 28$ and 4-1BB domains: pilot clinical trial results. Blood. 2012;119(17):3940-3950.

123. Zhou H, et al. The efficacy and safety of antiCD19/CD20 chimeric antigen receptor- T cells immunotherapy in relapsed or refractory B-cell malignancies:a meta-analysis. BMC Cancer. 2018;18(1):929.

124. Wang Y, et al. Effective response and delayed toxicities of refractory advanced diffuse large B-cell lymphoma treated by CD20-directed chimeric antigen receptor-modified T cells. Clin Immunol. 2014;155(2):160-175.

125. Ramos CA, et al. Clinical and immunological responses after $\mathrm{CD} 30$-specific chimeric antigen receptor-redirected lymphocytes. JClin Invest. 2017;127(9):3462-3471.

126. Wang CM, et al. Autologous T cells expressing $\mathrm{CD} 30$ chimeric antigen receptors for relapsed or refractory Hodgkin lymphoma: an open-label phase I trial. Clin Cancer Res. 2017;23(5):1156-1166.

127. Di Stasi A, et al. T lymphocytes coexpressing CCR4 and a chimeric antigen receptor targeting CD30 have improved homing and antitumor activity in a Hodgkin tumor model. Blood. 2009;113(25):6392-6402.

128. Mamonkin M, Rouce RH, Tashiro H, Brenner MK. A T-cell-directed chimeric antigen receptor for the selective treatment of T-cell malignancies. Blood. 2015;126(8):983-992.

129. Gomes-Silva D, et al. CD7-edited T cells expressing a CD7-specific CAR for the therapy of T-cell malignancies. Blood. 2017;130(3):285-296.

130. Maciocia PM, et al. Targeting the T cell receptor $\beta$-chain constant region for immunotherapy of $\mathrm{T}$ cell malignancies. Nat Med.2017;23(12):1416-1423.

131. Benjamin R, et al. Preliminary data on safety, cellular kinetics and anti-leukemic activity of UCART19, an allogeneic anti-CD19 CAR T-cell product, in a pool of adult and pediatric patients with high-risk CD19+ relapsed/refractory B-cell acute lymphoblastic leukemia. Blood. 2018;132(suppl 1):896.

132. Armand P, et al. Pembrolizumab in patients with relapsed or refractory primary mediastinal large B-cell lymphoma (PMBCL): data from the Keynote-013 and Keynote-170 studies. Blood. 2018;132(suppl 1):228.

133. Kwong YL, et al. PD1 blockade with pembrolizumab is highly effective in relapsed or refractory NK/T-cell lymphoma failing l-asparaginase. Blood.2017;129(17):2437-2442. 